Publ. RIMS, Kyoto Univ.

15 (1979), 741-747

\title{
Monodromy Structure of Solutions of Holonomic Systems of Linear Differential Equations is Invariant under the Deformation of the System
}

\author{
By \\ Masaki KASHIWARA ${ }^{(1)}$ and Takahiro KAWAI ${ }^{(2)}$
}

Inspired by some physical problems, Sato has recently proposed to investigate the deformation of systems of (micro-) differential equations with the emphasis on the invariance of global character of solutions. (Sato et al. [4]) Especially, he has observed that the classical result of Schlesinger [5] is most neatly explained from this point of view. The purpose of this note is to show that the monodromy structure of solutions of holonomic systems of linear differential equations is invariant under the deformation of the system specified below (Definition 1) on the condition that related topological structure does not change. (See Corollary 1 for the precise statement.)

We first list up some notations used in this note. We refer the reader to S-K-K [3] for notations not listed here.

$X$ : a complex maniford. A point in $X$ shall be denoted by $x$.

$\pi$ : the projection map from $T^{*} X$ to $X$.

$U:$ a connected Stein open set in $\boldsymbol{C}^{N}$. A point in $\boldsymbol{C}^{N}$ shall be denoted by $t=\left(t_{1}, \cdots, t_{N}\right)$.

$X_{c}=\{c\} \times X \subset U \times X$ for $c \in U$. The natural injection from $X_{c}$ to $U \times X$ shall be denoted by $\iota_{c}$. We sometimes identify $X_{c}$ with $X$.

Received August 1, 1977.

(1) Department of Mathematics, Massachusetts Institute of Technology. Current address: Research Institute for Mathematical Sciences, Kyoto University, Kyoto 606, Japan. Supported in part by NSF MCS 75-23334.

(2) Department of Mathematics, Harvard University and Research Institute for Mathematical Sciences, Kyoto University. Current address: Research Institute for Mathematical Sciences, Kyoto University, Kyoto 606, Japan.

Supported in part by NSF GP 36269. 
$S(c)$ : When $S$ is a subset of $U \times X, S(c)$ stands for $\{(t, x) \in S$; $t=c\}$.

$\widetilde{D}:$ the sheaf of linear differential operators of finite order defined on $X$ whose coefficients depend holomorphically on $t \in U$. For an element $P \in \widetilde{\mathscr{D}}_{x}, \frac{\partial P}{\partial t_{l}}$ stands for an operator obtained by differentiating the coefficients of $P$.

$\widetilde{\mathscr{M}}:$ a coherent left $\widetilde{\mathscr{D}}$-Module.

$\widetilde{\mathscr{M}}_{c}$ : the coherent left $\mathscr{D}_{X}$-Module ${ }^{(1)}$ obtained from $\widetilde{\mathscr{M}}$ by freezing $t=c$ in its coefficients. Its characteristic variety shall be denoted by $V(c)$.

$\pi_{1}(Z)$ : the fundamental group of a topological space $Z$.

The meaning of the deformation of the system investigated here is defined as follows. (See Kawai [1] and Sato et al. [4]. See also Lax [2].)

Definition 1. A coherent left $\widetilde{D}$-Module $\widetilde{\mathscr{M}}$ defined on $U \times X-S$ is said to enjoy a deformation property there if there exist a coherent $\mathscr{D}_{U \times X}$-Module $\mathscr{M}$ that is defined on $U \times X-S$ and a $\widetilde{D}$-linear homomorphism $H$ from $\widetilde{\mathscr{M}}$ to $\mathscr{M}$ so that they satisfy the following:

For any $c \in U, X_{c}-S(c)$ is non-characteristic with respect to $\mathscr{M}$ and $H$ induces an isomorphism from $\widetilde{\mathscr{M}}_{c}$ into the tangential system $\mathscr{M}_{X_{c}-S(c)}$ induced from $\mathscr{M}$ to $X_{c}-S(c)$ (S-K-K [3] Chapter II Definition 3. 5. 4).

Our main result is the following

Theorem 1. Assume that $X$ is compact. Assume that a coherent left $\widetilde{\mathscr{D}}$-Module $\widetilde{\mathscr{M}}$ is defined on $U \times X-S$ for a closed subset $S$ of $U \times X$ and enjoys the deformation property there. Assume that $\widetilde{\mathscr{M}}_{c}$ is a holonomic system, i.e., $V(c) \subset T^{*}\left(X_{c}-S(c)\right)$ is Lagrangian. Assume finally that $F \equiv S \cup\left\{(t, x) \in U \times X-S ; x \in \pi\left(V(t)-T_{X_{t}}^{*} X_{t}\right)\right\}$ defines a closed analytic subset of $U \times X$. Then, denoting by $i(c)$ the

(1) For the sake of simplicity of notations, we denote by $\mathscr{D}$ the sheaf of linear differential operators of finite order. In S-K-K [3] the notation $\mathscr{D}^{f}$ is used. 
natural map from $\pi_{1}(X-F(0))$ to $\pi_{1}(X-F(c)) \quad(|c| \ll 1)$ and denoting by $\rho_{c}$ the monodromy representation of $\pi_{1}(X-F(c))$ on the space $E_{c}$ of the multivalued analytic solutions of $\widetilde{\mathscr{M}}_{c}$, we have

$$
\rho_{0}=\rho_{c} \cdot i(c)
$$

for any $c$ in $U$.

Remark 1. The assumption that $X$ is compact and that $F$ is a closed analytic set guarantees the existence of the map $i(c)$.

Remark 2. Since $\widetilde{\mathscr{M}}_{c}$ is assumed to be a holonomic system of linear differential equations, the solutions to $\widetilde{\mathscr{M}}_{c}$, which are holomorphic outside $F(c)$, enjoy the finite determination property, i.e., $E_{c}$ is of finite dimension. Furthermore, as the proof of the theorem shows, $\mathscr{M}$ is also a holonomic system of linear differential equations, and hence $E_{c}$ are all isomorphic.

Proof of Theorem 1. Since $X_{c}$ is supposed to be non-characteristic with respect to $\mathscr{M}$,

$$
\rho: \operatorname{SS} \mathscr{M} \cap T^{*} X \rightarrow T^{*} X_{c}
$$

is a finite map. Therefore the holonomic character of $\widetilde{\mathscr{M}}_{c}=\mathscr{M}_{X_{c}}$ entails that $\mathscr{M}$ itself is holonomic. This implies that $\mathcal{M}$ is locally isomorphic to $\mathcal{O}_{\boldsymbol{V} \times X}^{m}$ for an integer $m$ outside $F$. Therefore, $\operatorname{Ham}\left(\mathscr{M}, \mathcal{O}_{U \times X}\right)$ defines a locally constant sheaf on $U \times X-F$. Furthermore, the CauchyKovalevsky theorem asserts

$$
\left.\operatorname{Ham}\left(\mathscr{M}, \mathcal{O}_{U \times X}\right)\right|_{X_{c}}=\operatorname{Ham}\left(\mathscr{M}_{X_{c}}, \mathcal{O}_{X}\right) \text {. }
$$

Note that this isomorphism is given through $F$. Therefore, by virtue of (2) combined with the fact that $\operatorname{Ham}\left(\mathscr{M}, \mathcal{O}_{U<X}\right)$ is locally constant outside $F$, we find the assertion of the theorem.

Q.E.D.

Corollary 1. Assume the same conditions as in Theorem 1. Assume furthermore that

$$
i(c): \pi_{1}(X-F(0)) \rightarrow \pi_{1}(X-F(c))
$$

is an isomarphism. Then the monodromy representation of solutions 
to $\widetilde{M}(c)$ is independent of $c$.

This corollary immediately follows from Theorem 1 .

Remark. Note that our results hold equally for equations with irregular singularities. In order to exemplify this fact, we present the following example.

Let us take $P^{1}(\boldsymbol{C})$ as $X$ and $\{t \in \boldsymbol{C} ;|t|<1\}$ as $U$ and let $S$ be the empty set. Let $\alpha$ be a complex number. Define $\widetilde{\mathscr{M}}$ by

$$
\left(\begin{array}{cc}
2 x \frac{\partial}{\partial x}-2 \alpha & -t \\
-t \frac{\partial}{\partial x} & 2 x \frac{\partial}{\partial x}-2 \alpha+1
\end{array}\right)\left(\begin{array}{l}
u_{1} \\
u_{2}
\end{array}\right)=\left(\begin{array}{l}
0 \\
0
\end{array}\right)
$$

and define $\mathscr{M}$ by

$$
\left\{\begin{array}{l}
\left(\begin{array}{cc}
2 x \frac{\partial}{\partial x}-2 \alpha & -t \\
-t \frac{\partial}{\partial x} & 2 x \frac{\partial}{\partial x}-2 \alpha+1
\end{array}\right)\left(\begin{array}{l}
u_{1} \\
u_{2}
\end{array}\right)=\left(\begin{array}{l}
0 \\
0
\end{array}\right) \\
\left(\begin{array}{ll}
\frac{\partial}{\partial t} & 1 \\
\frac{\partial}{\partial x} & \frac{\partial}{\partial t}
\end{array}\right)\left(\begin{array}{l}
u_{1} \\
u_{2}
\end{array}\right)=\left(\begin{array}{l}
0 \\
0
\end{array}\right) .
\end{array}\right.
$$

Then all the conditions posed in Theorem 1 are satisfied. It is clear that the solutions to equation $\mathscr{M}$ (or $\widetilde{\mathscr{M}}$ ) have essential singularities in general at $x=0$, still the monodromy structure of the solutions to equation $\widetilde{M}$ is invariant with respect to the parameter $t$.

Now that we have such a general result, we discuss one recipe due to Sato by which we can find $\mathscr{M}$. This recipe is a natural generalization of the recipe due to Lax [2].

For this purpose, we first consider $\boldsymbol{C}$-linear endomorphism $\Phi_{l}$ of $\widetilde{\mathscr{M}}$ defined on $U \times X-F(l=1, \cdots, N)$ that satisfy

$$
\Phi_{l} P u=P \Phi_{l} u+\frac{\partial P}{\partial t_{l}} u \quad(l=1, \cdots, N)
$$


for any $P \in \widetilde{\mathscr{D}}_{p}$, any $u \in \widetilde{\mathscr{M}}_{p}$ and any $p \in U \times X-F$,

$$
\Phi_{k} \Phi_{l}=\Phi_{l} \Phi_{k} \quad(l, k=1, \cdots, N) .
$$

Now we choose generators $u_{j}(j=1, \cdots, n)$ of $\widetilde{\mathscr{M}}$, that is,

$$
\widetilde{\mathscr{M}}=\sum_{j=1}^{n} \widetilde{\mathscr{D}} u_{j}
$$

In the sequel we denote by $u$ the column vector composed of $u_{j}(j=1$, $\cdots, n)$. Then we can find a matrix of linear differential operators $X^{(l)}$ $=\left(X_{j, k}^{(l)}\right)_{1 \leq j, k \leq n}$ with $X_{j, k}^{(l)} \in \widetilde{\mathscr{D}}$ such that

$$
\Phi_{l} u_{j}=\sum_{k=1}^{n} X_{j, k}^{(l)} u_{k} \quad(j=1, \cdots, n, \quad l=1, \cdots, N)
$$

holds. Note that $\Phi_{l}$ does not determine $X^{(l)}$ uniquely even after we fix the representation (5) of $\widetilde{\mathscr{M}}$.

Now we define the required system $\mathscr{M}$ by adding following equations

$$
\frac{\partial}{\partial t_{l}} U=X_{l} U \quad(l=1, \cdots, N)
$$

to the equation $\widetilde{M}$, that is, we define $\mathscr{M}$ by

$$
\left(\mathscr{D}{ }_{\times x} \underset{\mathscr{\mathscr { D }}}{\otimes} \widetilde{\mathscr{M}}\right) /\left(\sum_{l=1}^{N} \mathscr{D}_{U \times X}\left\{\frac{\partial}{\partial t_{l}} \underset{\widetilde{\mathscr{D}}}{\otimes} U-1 \underset{\mathscr{D}}{\otimes} \Phi_{l} U ; U \in \widetilde{\mathscr{M}}\right\}\right) .
$$

We now assume that any $X_{c}$ is non-characteristic with respect to the equation (8).

Then we have the following

Theorem 2. The system $\mathscr{M}$ defined by (8) satisfies

$$
\widetilde{\mathscr{M}} /\left(\sum_{l=1}^{N}\left(t_{l}-c_{l}\right) \widetilde{\mathscr{M}}\right) \widetilde{M} /\left(\sum_{l=1}^{N}\left(t_{l}-c_{l}\right) \mathscr{M}\right)
$$

for any $c=\left(c_{1}, \cdots, c_{N}\right) \in U$, namely, the induced system $\mathscr{M}_{X_{c}}$ coincides with $\widetilde{\mathscr{M}}_{c}$.

Proof. We may assume without loss of generality that $c=0$. We prove the theorem by the induction on $N$. We first discuss the case where $N=1$. In this case, it suffices to show that the following natural map 


$$
j: \widetilde{\mathscr{M}} / t_{1} \widetilde{\mathscr{M}} \rightarrow \mathscr{M} / t_{1} \mathscr{M}
$$

is an isomorphism. Since it is clear that $j$ is surjective, it is enough to prove that $j$ is injective.

We first choose a $d \times n$ matrix $\widetilde{P} \in M(d, n ; \widetilde{D})$ such that $\widetilde{P} u=0$ and that any annihilator $\left(\widetilde{Q}_{1}, \cdots, \widetilde{Q}_{n}\right)$ of $u$ is written as $\widetilde{T} \widetilde{P}$ for some $\widetilde{T}$ $\in M(1, d ; \widetilde{D})$. Let $w$ be an element of $\widetilde{\mathscr{M}}$ such that $j(w)=0$ in $\mathscr{M} / t_{1} \mathscr{M}$. We choose $\tilde{A}_{j}$ so that $w$ is written as $\sum_{j=1}^{N} \tilde{A}_{j} u_{j}$ and define $\tilde{A}$ as the row vector $\left(\tilde{A}_{1}, \cdots, \tilde{A}_{n}\right)$. Then we have

$$
\widetilde{A}=B \widetilde{P}+R\left(\frac{\partial}{\partial t_{1}}-X_{1}\right)+t_{1} G
$$

with $B \in M(1, d ; \widetilde{D}), R \in M(1, n ; \mathscr{D})$ and $G \in M(1, n ; \mathscr{D})$.

On the other hand, in view of condition (3), we can find a $d \times d$ matrix $Y_{1} \in M(d, d ; \widetilde{\mathscr{D}})$ such that

$$
\widetilde{P}\left(\frac{\partial}{\partial t_{1}}-X_{1}\right)=\left(\frac{\partial}{\partial t_{1}}-Y_{1}\right) \widetilde{P} .
$$

Furthermore, by virtue of the Späth type theorem for differential operators, we can find a matrix $\widetilde{B} \in M(1, d ; \widetilde{D})$ such that

$$
B=\widetilde{B}+S\left(\frac{\partial}{\partial t_{1}}-Y_{1}\right)
$$

for a matrix $S \in M(1, d ; \mathscr{D})$. The same argument also applies to $G$. Therefore we have

$$
\widetilde{A}-\widetilde{B} \widetilde{P}-t \widetilde{G}=R^{\prime}\left(\frac{\partial}{\partial t_{1}}-X_{1}\right)
$$

where $\widetilde{B}$ and $\widetilde{G}$ belong to $M(1, d ; \widetilde{D})$ and $M(1, n ; \widetilde{D})$, respectively. Then, the uniqueness assertion in the Späth-type theorem quoted above implies that $R^{\prime}=0$. This means that $\tilde{A} u=t_{1} \widetilde{G} u$ belongs to $t_{1} \widetilde{\mathcal{M}}$. Thus we have shown that $j$ is injective. This completes the proof of the theorem in the case where $N=1$. Next we show the theorem in the general case. In order to simplify the notations, we discuss the case $N=2$. The argument for the general case does not require any essential change. In order to prove the theorem in this case, we define another equation $\widetilde{\mathscr{M}}$. by adding only 


$$
\frac{\partial}{\partial t_{1}} U=X_{1} U
$$

to $\mathscr{M}$, namely

$$
\widetilde{\mathscr{M}}_{1}=\left(\underset{\mathscr{D}}{\widetilde{\mathscr{D}}_{1}} \underset{\widetilde{\mathscr{D}}}{\otimes} \widetilde{\mathscr{M}}\right) /\left(\widetilde{\mathscr{D}}_{1}\left\{\frac{\partial}{\partial t_{1}} \underset{\widetilde{\mathscr{D}}}{\otimes} U-1 \otimes \widetilde{\Phi}_{1} U ; U \in \widetilde{\mathscr{M}}\right\}\right),
$$

where $\widetilde{D}_{1}$ denotes the sheaf of the linear differential operators in $t_{1}$ and $x$ which depend holomorphically on $t_{2}$. Next define $\Phi_{2}^{\prime}$ by extending the action of $\Phi_{2}$ on $\widetilde{M}$ so that

$$
\Phi_{2}(P \otimes U)=P \otimes \Phi_{2} U+\frac{\partial P}{\partial t_{2}} \otimes U
$$

holds for any $U \in \widetilde{\mathscr{M}}$ and $P \in \widetilde{\mathscr{D}}_{1}$. Then clearly $\widetilde{\Phi}_{2}$ defines a section of $\operatorname{End}_{\boldsymbol{C}}\left(\widetilde{\mathscr{M}}_{1}\right)$. Then it suffices to show that

$$
\frac{\partial P}{\partial t_{2}}=\Phi_{2} P-P \Phi_{2}
$$

holds for any $P \in \widetilde{\mathscr{D}}_{1}$ as an equality in $\operatorname{End}_{\boldsymbol{c}}\left(\widetilde{\mathscr{M}}_{1}\right)$. The relation (17) is, however, a direct consequence of (4).

Q.E.D.

\section{References}

[1] Kawai, T., Invariance of cohomology groups under a deformation of an elliptic system of linear differential equations. Proc. Japan Acad. 53A (1977), 144-145.

[2] Lax, P. D., Intgrals of non-linear equations of evolutions and solitary waves. Comm. Pure Appl. Math. 21 (1968), 467-490.

[3] Sato, M., Kawai, T. and Kashiwara, M. (Referred to as S-K-K [3]): Microfunctions and pseudo-differential equations, Springer's Lecture Notes in Mathematics No. 287, pp. 265-529, Berlin-Heidelberg-New York, Springer, 1973.

[4] Sato, M., Miwa, T. and Jimbo, M.: Studies on holonomic quantum fields. II, Proc. Japan Acad. 53A (1977) 147-152.

[5] Schlesinger, L., Über eine Klasse von Differential Systemen beliebiger Ordnung mit festen kiritischen Punkten. J. Reine Angerw. Math. 141, 96-145 (1912). 
